\title{
Sliding Mode Control for Diesel Engine Using Extended State Observer
}

\author{
Haoping WANG, Wei ZHANG, Yang TIAN*, Qiankun QU \\ Nanjing University of Science \& Technology, School of Automation, \\ Sino-French International Joint Laboratory of Automatic Control and Signal Processing (LaFCAS), \\ Nanjing 210094, CHINA \\ hp.wang@njust.edu.cn; 250658559@qq.com; tianyang@njust.edu.cn; qqkiller61@163.com \\ * Corresponding author
}

\begin{abstract}
This paper does a research on turbocharged diesel engines both of air-path or speed-path alone, and proposes a cooperative control strategy of air and speed-path two loop TDE system. For modern diesel engines, accurate air/fuel ratio (AFR) and exhaust gas recirculation (EGR) rates design are very important to meet the requirements of emission standards of NOx and PM. For the EGR and AFR rates are controlled by the EGR and the variable geometry turbine (VGT) actuators, we propose a fourth-order simplified nonlinear model which takes into account the crankshaft speed dynamics and the air-path dynamics for the turbocharged diesel engine. The controller for the speed-path we designed which is based on Lyapunov function is used to track the desired engine speed. For the air-path, a sliding mode controller based on exponential reaching law which uses the concept of total disturbances to control the EGR and VGT valve is designed. In order to estimate the disturbances in the system, we proposed a 2nd-order extended space observer (ESO). The simulation results show that the sliding mode controller which utilizes observer and reaching law can alleviate the chattering problem obviously and the proposed two-loop structure dynamic model exhibits excellent performances in tracking desired signals and overcoming the system disturbances. In the presence of disturbances, the system can still observe the total disturbances, including the unmodeled dynamics and actuator faults, accurately and timely.
\end{abstract}

Keywords: Turbocharged diesel engine; sliding mode control; ESO; chattering; tracking; disturbances.

\section{Introduction}

With the rapid development of vehicles in transportation, the exhaust emissions increase seriously the pollution of our environment. The energy efficiency and the environment protection become the prominent concerns in transportation, and make people pay more and more attention to them. How to improve vehicle fuel economy and exhaust emissions standards in order to save energy and reduce pollution is now an extremely urgent problem. Therefore, energy-saving technology of a car engine is one of the trends in the automotive future.

Comparing to gasoline engines, diesel engines have the advantage of providing larger torque under a compromise between fuel consumption and given exhaust emission level. However, diesel engines also have some problems to be resolved and one prominent question is exhaust emissions. Although the emissions of diesel engines in $\mathrm{CO}$, hydrocarbons $(\mathrm{HC})$ and $\mathrm{CO}_{2}$ is lower than gasoline engines, but the emissions of particulate matter (PM) and nitrogen oxides (NOx) are difficult to control.

In order to meet the requirements of emission standards EURO V and VI, especially for PM and NOx emissions, the engine needs to be controlled in each cycles.

Earlier reduction mechanism suggest that NOx emissions can be reduced by increasing the exhaust gas recirculation (EGR) intake manifold fraction and smoke can be reduced by increasing the air/fuel ratio (AFR) [1]. The EGR and AFR ratio is controlled by a variable geometry turbine (VGT) actuator. So, how to design the controller to control the VGT position for the purpose of reducing emissions of NOx and the smoke has become the continuous studies of researchers in recent years.

At present, many foreign researchers have done a lot of work on diesel engine control strategy and has made some progress. However, the EGR and AFR actuators are strongly coupled so that the traditional PI regulator face difficult in providing satisfactory results in the terms of torque response, transient response and engineout emissions even with time- consuming and detailed calibrations.

In the past decade, many diesel engine (TDE) air and speed-path model are in constant development. In [2], the authors proposed a full-seven-order TDE air-path model. The model describes the dynamics of several variables such as the pressure and the oxygen 
mass fractions in the intake and exhaust manifolds, the turbocharger speed, and the two states of the control signals which describe the dynamics of the EGR and VGT actuator. To simplify the control design, the full-seven-order TDE model was simplified into a third-order one. But this simplification resulted in neglecting the unmodeled dynamics, system disturbances or the engine faults affecting the air-path.

With the development of digital processing technology, many sophisticated control strategies are introduced in controlling modern diesel engines. For speed engine regulation, some prior efforts already exist, such as optimal gain scheduling [3], $\mathrm{H} \infty$ control based on linear parameter varying (LPV) approach [4], adaptive methods [5], sliding mode control [6], Lyapunov function based control [7], or PID control [8]. And for the air-path, several controllers were proposed in the literature, for example, Lyapunov control design [2], robust gain-scheduled controller based on a LPV model for turbocharged diesel engine [9], indirect passivation [10], and feedback linearization [11].

However, most of these algorithms are oriented model, that is, the designed control laws are based on the model of diesel engines. In fact, actuator failure in the input control, can be regarded as external disturbances; modeling uncertainties which act on the system from different channels can be regarded as internal disturbances. Meantime, the total disturbances of the system are difficult to measure, thus, a disturbance observer is needed to estimate them.
So one of the main purpose of this paper is to design a control strategy which is robust when facing the disturbances which affects the diesel engine air and speed-path. Here, a sliding mode controller based on extended space observer (ESO) is chosen for the control design [12-14]. In addition, the existing studies are made for airpath or speed-path respectively [12-15]. For the reason that the two paths influence each other deeply, we desire to establish a two loop cooperative control system.

This paper is organized as follows. Section 2 presents the TDE air and speed-path modeling and the faulty system that we will deal with in this paper. Section 3 develops the controller for the two loop TDE system, the Lyaounov based controller for the speed-path and the ESO based sliding mode controller for the air-path. Then we show some numerical simulation results in Section 4. Finally, some concluding remarks and discussions are given in Section 5.

\section{Modeling of Turbocharged Diesel Engine}

\subsection{TDE model description}

The schematic diagram of TDE which including the EGR, VGT, and crankshaft [16] is illustrated in Figure 1.

At the bottom of the diagram, we can see that the compressor and the VGT are mounted on the same crank shaft. During the engine running, the turbine takes the energy from the exhaust gas to power the compressor. Meantime, the fresh air brought in via the

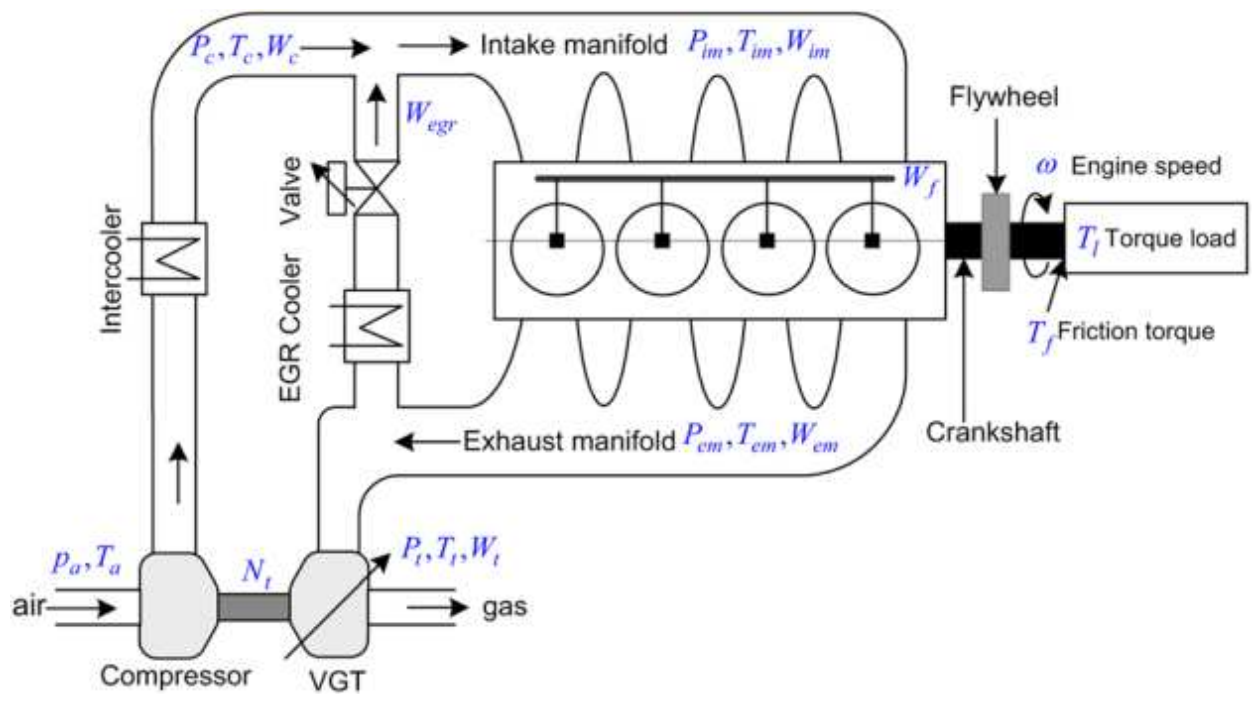

Figure 1. Turbocharged diesel engine diagram. 
intercooler and the exhaust gas from the EGR valve mix together, then burn with the injected fuel in the cylinder and produce torque on the crankshaft. But the diesel engine belongs to the intermittent combustion power unit, the energy from the cylinder transmitted to the crankshaft is not stable and continuous. Therefore, we add a flywheel to the crankshaft, and then the power transmitted to the crankshaft during the power stroke was stored to ensure the output torque of the crankshaft angular velocity as uniformly as possible, and make it possible to overcome the engine overload in a short time.

\subsection{TDE mathematical modeling}

Diesel engine air-path dynamic subsystem: The full-order TDE air-path model is a seventhorder one which contains the intake and exhaust manifold pressure $\left(p_{i m}\right.$ and $\left.p_{e m}\right)$, oxygen mass fractions in the intake and exhaust manifold $\left(F_{i m}\right.$ and $\left.F_{e m}\right)$, and etc.

In order to simplify the model and to get a simple control law, also for the fact that the oxygen mass fraction is difficult to mearsure, the seventh-order model is reduced to a third one [2].

$$
\begin{aligned}
& \dot{p}_{i m}=k_{i m}\left(W_{c}+W_{e g r}-W_{i e}\right) \\
& \dot{p}_{e m}=k_{e m}\left(W_{i e}+W_{f}-W_{e g r}-W_{t}\right) \\
& \dot{P}_{c}=\frac{1}{\tau}\left(\eta_{m} P_{t}-P_{c}\right)
\end{aligned}
$$

where Pc represents the power of the compressor. The mass flow rate of the compressor and the turbocharger is related to the power as follows:

$$
W_{c}=P_{c} \frac{k_{c}}{\left(p_{i m} / p_{a}\right)^{\mu}-1}
$$

and $P_{t}=k_{t}\left(1-\left(p_{e m} / p_{a}\right)^{-\mu}\right) W_{t}$

The flow rate from the intake manifold to the engine cylinder $W_{i e}(\mathrm{Kg} / \mathrm{s})$ can be modeled as

$$
W_{i e}=k_{e} p_{i m}
$$

with $k_{e}=\frac{\eta_{v} \omega V_{d}}{4 \pi R T_{i m}}$, where $\eta_{v}$ is used to describe the volume ratio of the engine, and can be represented approximately as [17].

$$
\eta_{v}=\alpha_{0}+\alpha_{1} \omega+\alpha_{2} \omega^{2}
$$

With $\alpha_{0}, \alpha_{2}>0, \alpha_{1}<0$. The coefficient in (1) (3) can be represented as follows:

$$
\begin{array}{ll}
k_{c}=\frac{\eta_{c}}{c_{p} T_{a}}, & k_{t}=c_{p} \eta_{t} T_{e m} \\
k_{i m}=\frac{R T_{i m}}{V_{i m}}, & k_{e m}=\frac{R T_{e m}}{V_{e m}}
\end{array}
$$

1) Diesel engine speed-path dynamic subsystem: According to the Newton's second law, the dynamics of the crankshaft system can be described as

$\dot{\omega}=\frac{1}{J \omega}\left(P_{e}-P_{f l}\right)$

where $\omega(\mathrm{rad} / \mathrm{s})$ is the engine speed, $\mathrm{J}(\mathrm{Kg} / \mathrm{m} 2)$ is the inertia of the crankshaft and can be regarded as a constant. $P_{e}(\mathrm{~W})$ is the effective mechanical power of the engine, $P_{f l}(\mathrm{~W})$ represents the friction and load power. $P_{e}$ can be defined as follows:

$P_{e}=H_{f} \eta_{e} W_{f}$

where $H_{f}(J / K g)$ is on behalf of the calorific value of the fuel, $\eta_{e}$ is the effective conversion efficiency of the engine, and $W_{f}(\mathrm{Kg} / \mathrm{s})$ is the fuel flow rate.

$\eta_{e}$ can be expressed in different forms, which can be represented by two equations [16]. In this paper, our model is selected as

$$
\eta_{e}=a_{\lambda}+b_{\lambda} \lambda+c_{\lambda} \lambda^{2} \text {, and } \lambda=W_{i e} / W_{f}
$$

2) The TDE model analysis: Thus, the TDE air and speed-path model can be denoted as the following control-affine form:

$$
\begin{aligned}
& \dot{x}=f\left(x, u_{t}\right)+g(x) u \\
& \dot{\omega}=q_{1}\left(\omega, p_{i m}\right) u_{1}+q_{2}\left(\omega, p_{i m}\right)+\frac{q_{3}\left(\omega, p_{\text {im }}\right)}{u_{1}}
\end{aligned}
$$

with $u_{1}=W_{f}, u_{2}=W_{e g r}, u_{3}=W_{t}, x=\left[p_{i m}, p_{e m}, P_{c}\right]^{T}$, $u=\left[u_{2}, u_{3}\right]^{T}$,

$q_{1}\left(\omega, p_{i m}\right)=\frac{H_{f} a_{\lambda}}{J \omega}$,

$q_{2}\left(\omega, p_{\text {im }}\right)=$

$=\frac{1}{J \omega}\left(\frac{H_{f} b_{\lambda} V\left(\alpha_{0}+\alpha_{1} \omega+\alpha_{2} \omega^{2}\right) \omega p_{i m}}{4 \pi R T_{i m}}-P_{f l}\right)$,

$q_{3}\left(\omega, p_{i m}\right)=\frac{H_{f} c_{\lambda} V_{d}^{2}\left(\alpha_{0}+\alpha_{1} \omega+\alpha \omega^{2}\right)^{2} \omega p_{i m}^{2}}{16 J \pi^{2} R^{2} T_{i m}^{2}}$, 


$$
\begin{gathered}
f\left(x, u_{1}\right)=\left[\begin{array}{cc}
k_{i m} k_{c} \frac{P_{c}}{\left(p_{i m} / p_{a}\right)^{\mu}-1}-k_{i m} k_{e} p_{i m} \\
k_{e m}\left(k_{e} p_{i m}+u_{1}\right) \\
\frac{-P_{c}}{\tau}
\end{array}\right], \\
g(x)=\left[\begin{array}{cc}
k_{i m} & 0 \\
-k_{e m} & -k_{e m} \\
0 & K_{0}\left(1-\left(p_{e m} / p_{a}\right)^{-\mu}\right)
\end{array}\right] .
\end{gathered}
$$

Assumption 1. System (10) and (11) are locally reachable.

Assumption 2. Considering that in system (10), time-based actuator faults are added in some way, then the faulty air-path model can be written as

$$
\dot{x}=f\left(x,\left(u_{1}+F_{1}(x, t)\right)\right)+g(x)(u+F(x, t))
$$

where $F_{1}(x, t), F(x, t)$ converges to an unknown positive constant $D_{m 1}, D_{m 2}$ respectively; that is,

$$
\begin{aligned}
& \left\|F_{1}(x, t)\right\|<D_{m 1} \\
& \|F(x, t)\|<D_{m 2}
\end{aligned}
$$

where $F_{1}$ represents the fuel valve fault, $F=\left(F_{2}\right.$, $F_{3}$ ) represents a actuator failure from the EGR and VGT valve respectively. In the physical sense, these faults may be caused by the error of the valve opening.

Remark 3. Noted that in [2], the authors proved that the set $\Omega$ which defined as

$$
\left\{\begin{array}{l}
\Omega=\left(p_{i m}, p_{e m}, P_{c}\right): \begin{array}{r}
1 \\
1<p_{i m}<p_{i m}^{\max }<p_{e m}^{\max } \\
1
\end{array}, P_{c}<P_{c}^{\max }
\end{array}\right\}
$$

is a invariant set. It means that for all initial conditions $\quad x(t 0) \in \Omega$, then we have $x(t) \in \Omega, \quad \forall t>t 0$.

3) Control Objective and Problem Statement: In this paper, we need to design the controller which can overcome the system disturbances that take into account the actuator failure such as the EGR and VGT valve faults. For inner loop, that is, for speed-path, considering system (11), we desire to design a continuous and stable control law, so that the engine speed is able to track the reference speed we want, that means, $\omega_{d} \rightarrow \omega$. In order to meet the nominal performance of the system, the suitable variable outputs is needed. And for outer air-path loop, the best vector output is

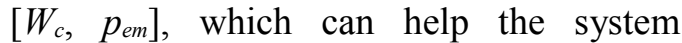
achieve optimal control performance. However, the differential equation of state variable $W_{c}$ is rather complex. In [18], the authors proposed a coordinate transformation to replace $W_{c}$ with $p_{i m}$ via considering the relation (2):

$p_{i m}=p_{a}\left(\frac{k_{c} P_{c}}{W_{c}}+1\right)^{\frac{1}{\mu}}$

that is,

$p_{\text {imd }}=p_{a}\left(\frac{k_{c} P_{c}}{W_{c d}}+1\right)^{\frac{1}{\mu}}$

By choosing new vector coordinates $\left(p_{i m}, p_{e m}\right)$, we can greatly simplify the control structure. Now, we consider the system (12) by defining the following two sliding variables:

$S_{1}=p_{\text {im }}-p_{\text {imd }}$,
$S_{2}=p_{\text {em }}-p_{\text {emd }}$

According to (10) (12), considering the faulty air-path diesel engine system, the time derivative of $S_{1}, S_{2}$ can be expressed as:

$$
\begin{aligned}
& \dot{S}_{1}=k_{i m} k_{c} \frac{P_{c}}{\left(p_{i m} / p_{a}\right)^{u}-1}+k_{\Im} u_{2}- \\
& \quad-k_{i m} k_{e} p_{i m}+k_{i m} F_{2}-\dot{p}_{\text {imd }}, \\
& \dot{S}_{2}=k_{e m} d_{e} p_{i m}+k_{e m} u_{1}-k_{e m} u_{2}-k_{e m} u_{3}+ \\
& \quad+k_{e m}\left(F_{1}-F_{2}-F_{3}\right)-\dot{p}_{e m d}
\end{aligned}
$$

Thus, the control objective of outer loop can be stated as follows: considering the faulty system (12) and the sliding variable $S=\left(S_{1}, S_{2}\right)^{T}$, our purpose is to find a effective control law $u=\left[u_{2}, u_{3}\right]^{T}$ so that the sliding variable $S$ converges to zero in a finite time.

\section{Control Law Design for Two- loop System}

The schematic diagram of TDE air and speedpath two loop system is shown in Figure 2.

We can see from the diagram that the inner loop transfers parameters $\mathrm{u} 1$ and $\omega$ to the outer loop, and the outer loop transfers $p_{\text {im }}$ to the inner one. The feedback dynamic control system consists of an inner loop of Lyapunov function based controller and an outer loop of sliding mode controller based on ESO. The inner one is designed to tracking the desired engine speed, and the outer one to ensure the 


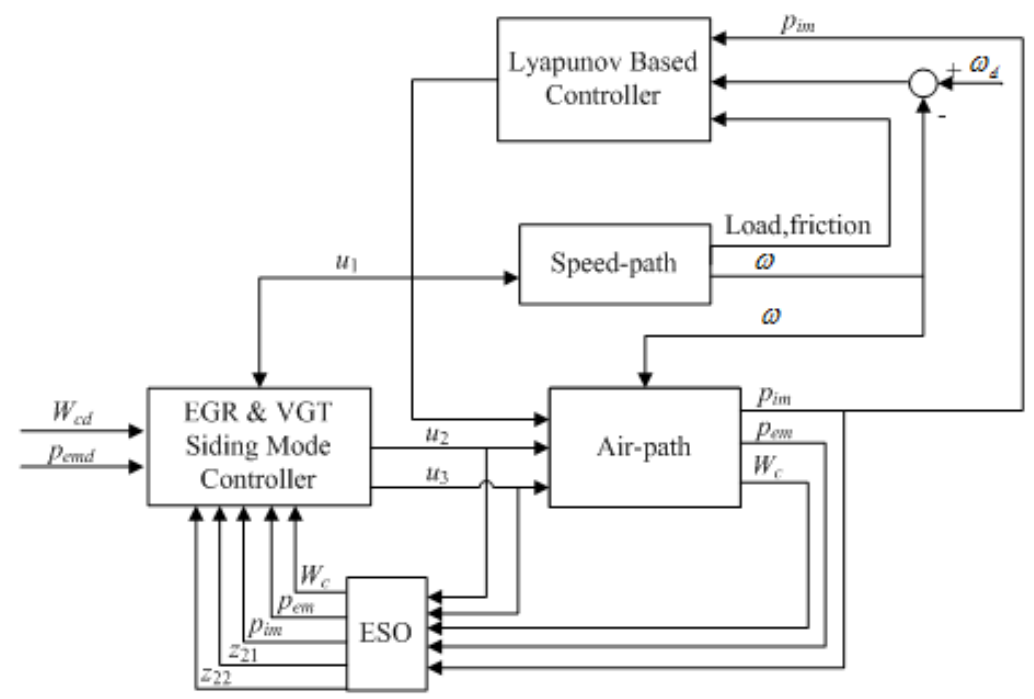

Figure 2. Air and speed-path two loop system schematic diagram.

desired control variables which can meet the emission performance.

\subsection{Lyapunov function based controller for inner loop}

Consider the engine speed dynamic system, we design a stabilization controller which is based on Lyapunov function. The Lyapunov function we choose is shown as

$$
V(\omega)=\frac{1}{2} e_{\omega}^{2}, \text { with } e_{\omega}=\omega_{d}-\omega
$$

Obviously, $V(\omega) \geq 0$.

In order to make ensure that its derivation is negative, that is:

$$
\dot{V}(\omega)=-e_{\omega} \dot{\omega}<0
$$

We choose the following function to stabilize the dynamic system:

$$
\dot{\omega}=K \cdot e_{\omega} \text {, with } K>0
$$

So, due to the Lyapunov function is positive and its derivation is negative, we can ensure that the inner loop system is asymptotically stable.

From (11) and (19), we obtains

$$
\begin{array}{r}
q_{1}\left(\omega, p_{\text {im }}\right) u_{1}+q_{2}\left(\omega, p_{\text {im }}\right)+\frac{q_{3}\left(\omega, p_{\text {im }}\right)}{u_{1}}= \\
=K \cdot e_{\omega}
\end{array}
$$

where $q_{1}\left(\omega, p_{i m}\right)>0$ and $q_{3}\left(\omega, p_{\text {im }}\right)<0[7,16]$.

Then, the algebraic expression of the fuel rate $u_{1}(t)$ can be calculated as

$$
u_{1}=\frac{-\left(q_{2}\left(\omega, p_{\text {im }}\right)-K \cdot e_{\omega}\right)+\sqrt{\delta}}{2 q_{1}\left(\omega, p_{\text {im }}\right)}
$$

where

$$
\begin{aligned}
\delta & =\left[q_{2}\left(\omega, p_{i m}\right)-K \cdot e_{\omega}\right]^{2}- \\
& -4 q_{1}\left(\omega, p_{i m}\right) \cdot q_{3}\left(\omega, p_{i m}\right) .
\end{aligned}
$$

For the fact of $q_{1}\left(\omega, p_{i m}\right)>0$ and $q_{3}\left(\omega, p_{i m}\right)<0$, we can ensure that the fuel rate $u_{1}(t)$ is always a real positive variable, which is consistent with the physical meaning of the actual system.

\subsection{ESO based sliding mode controller for outer loop}

For the outer air-path loop, our purpose is to stabilize the faulty system (12) in a finite time. That is, to find a suitable control law so that the sliding variables $S$ converges to zero in a finite time.

Let we rewrite the faulty system (12) as the following formula:

$$
\dot{S}=f_{0}(x)+g_{0}(x) u+\widetilde{d}-\dot{S}_{d}
$$

where

$$
\begin{aligned}
& S=\left[\begin{array}{l}
S_{1} \\
S_{2}
\end{array}\right], \\
& f_{0}=\left[\begin{array}{l}
k_{i m} W_{c}-k_{i m} k_{e} p_{i m} \\
k_{e m} k_{e} p_{i m}+k_{e m} u_{1}
\end{array}\right], \\
& g_{0}=\left[\begin{array}{rr}
k_{i m} & 0 \\
-k_{e m} & -k_{e m}
\end{array}\right],
\end{aligned}
$$


$\dot{S}_{d}=\left[\begin{array}{c}\dot{p}_{\text {imd }} \\ \dot{p}_{\text {emd }}\end{array}\right]$,

$\widetilde{d}=\left[\begin{array}{c}k_{i m} F_{2} \\ k_{e m}\left(F_{1}-F_{2}-F_{3}\right)\end{array}\right]$.

To alleviate the common chattering problem in sliding mode control, we choose the following exponential reaching law which achieves a good dynamic quality:

$$
\dot{S}=-\gamma S-\lambda \operatorname{sgn} S
$$

where $-\gamma S$ is the exponential part, its algebraic solution is $s(t)=s(0) \exp (-\gamma \cdot t)$, where $s(0)$ is the initial value of $s$. We can find that if $\gamma$ is chosen large enough, the system state will move to the switching surface with greater speed when $s$ is too large, and when $s$ is small, we can reduce the parameter $\lambda$ to inhibit the chattering.

Combing (22) and (23), the mathematical expressions of the control law $\mathrm{u}$ can be calculated as follows

$$
u=g_{0}^{-1}(x)\left(-\gamma S-\lambda \operatorname{sgn} S-f_{0}(x)-\widetilde{d}+\dot{S}_{d}\right)
$$

where the total disturbances of the system $\tilde{d}$ is entirely unknown so that it cannot be applied to the actual system. We notice that the control law above can be divided in two parts, one is totally known and the other is completely unknown.

For the purpose of estimating the disturbances and apply it to the actual system, we propose an ESO to observe it.

In (22), we introduce an extended state $X$ to observe the total disturbances $\widetilde{d}$. Then, we can obtain the following augmented system:

$$
\begin{aligned}
& \dot{S}=f_{0}(x)+g_{0}(x) u+X-\dot{S}_{d} \\
& \dot{X}=h(t)
\end{aligned}
$$

where $h(t)$ represents the derivative of the disturbances $\widetilde{d}$.

We proposed the following ESO [12]:

$$
\begin{aligned}
& E_{1}=Z_{1}-S \\
& \dot{Z}_{1}=Z_{2}+f_{0}+g_{0} u-\dot{S}_{d}-\beta_{1} E_{1} \\
& \dot{Z}_{2}=-\beta_{2}\left|E_{1}\right|^{\frac{1}{2}} \operatorname{sgn}\left(E_{1}\right)
\end{aligned}
$$

where $E_{1} \in R^{2}, Z_{1} \in R^{2}$ and $Z_{2} \in R^{2} . \beta_{1}$ and $\beta_{2}$ are the constant gains of ESO. $E_{1}$ represents the error of the observer, and $Z_{1}, Z_{2}$ are the two states of ESO. $Z_{1}$ is used to track the sliding variable $S$ and $Z_{2}$ is used to estimate the disturbances $\widetilde{d}$.

The dynamic error differential equation of systems (25-26) can be derived as

$$
\begin{aligned}
& \dot{E}_{1}=Z_{2}-\beta_{1} E_{1}-X=-\beta_{1} E_{1}+E_{2} \\
& \dot{E}_{2}=\dot{Z}_{2}-h(t)=-\beta_{2}\left|E_{1}\right|^{\frac{1}{2}} \operatorname{sgn}\left(E_{1}\right)-h(t)
\end{aligned}
$$

Obviously, the system (27) is stable in finite time ([18]).

We notice that the external and internal disturbances $\widetilde{d}$ can be estimated by $Z_{2}$ in the limited time, then, we can compensate it in the feedback loop.

As the total disturbances $\widetilde{d}$ are estimated as the system operating, we obtain the final control law:

$u=g_{0}^{-1}(x)\left(-\gamma S-\lambda \operatorname{sgn} S-f_{0}(x)-Z_{2}+\dot{S}_{d}\right)$

Combining (25) with (28), we obtain the following system:

$$
\dot{S}=-\gamma S-\lambda \operatorname{sgn} S-E_{2}
$$

and the sliding surface $S$ will converge to 0 in finite time [12].

\section{Numerical Simulation Results}

In this paper, the engine we choose is a common-rail direct-injection inline fourcylinder diesel engine produced by a French manufacturer. In order to illustrate the tracking performance and the immunity performance of the two loop system, the added friction and load power is shown in Figure 3.

The parameters of the model are introduced in [20]. The discontinuous sign function can be replaced by the following approximations: $\operatorname{sgn}(s)=\frac{s}{|s|+\xi}$ with $\xi=0.01$. In the two loop system, the numerical simulations are implemented under the following parameters: $K_{\omega}=20, \lambda=5, \gamma=150, \beta_{11}=\beta_{12}=5000, \beta_{21}=900000$, $\beta_{22}=500000$. In the simulation, the added actuator faults are square waves with amplitude of 0.01 and period of 10 seconds. The response of desired speed and actual speed are shown in Figure 4. And the response of control variables are shown in Figure 5 which illustrates its good tracking performance. 


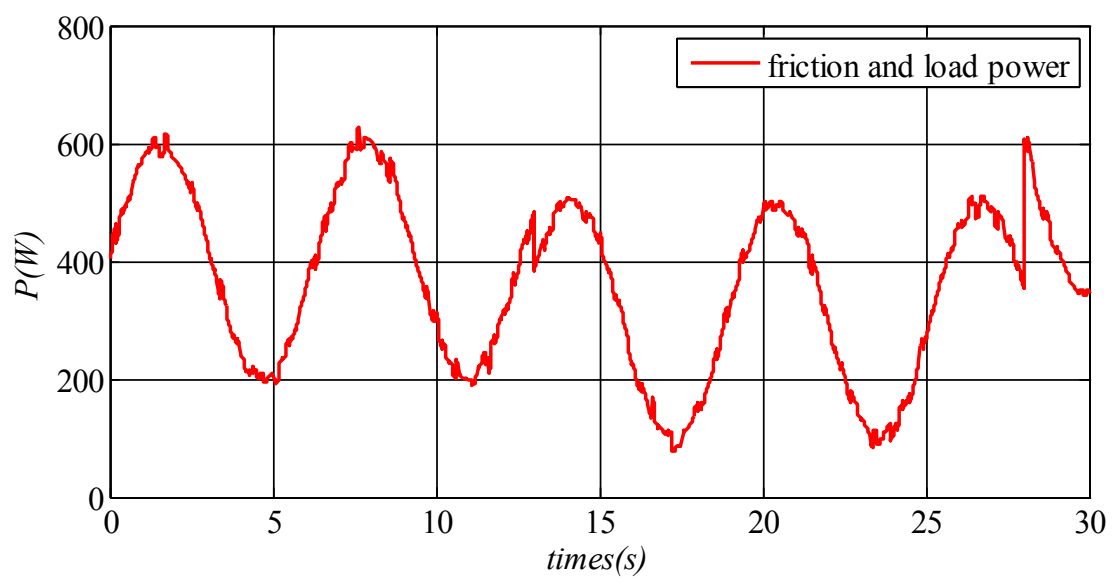

Figure 3. External friction and load power

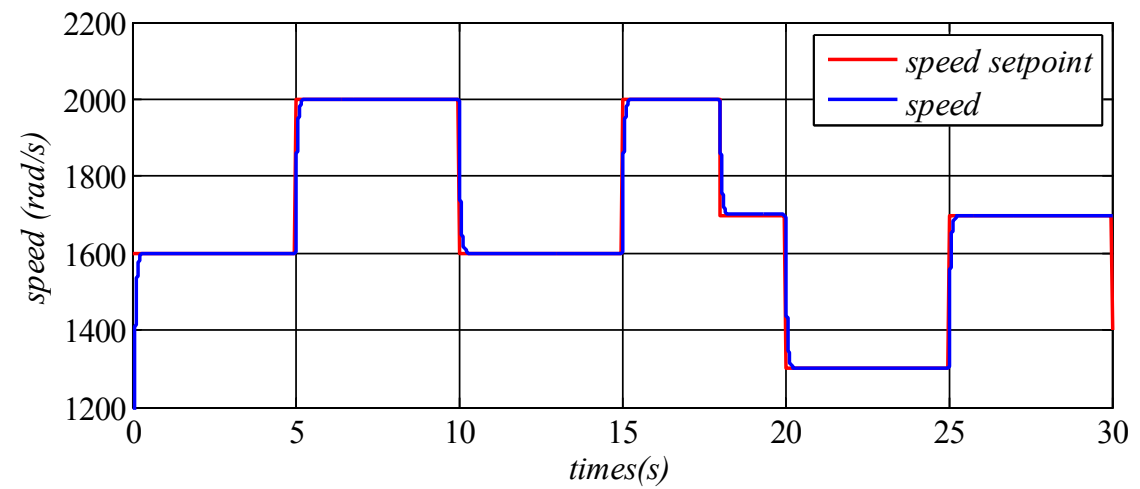

Figure 4. Engine speed $\omega$.
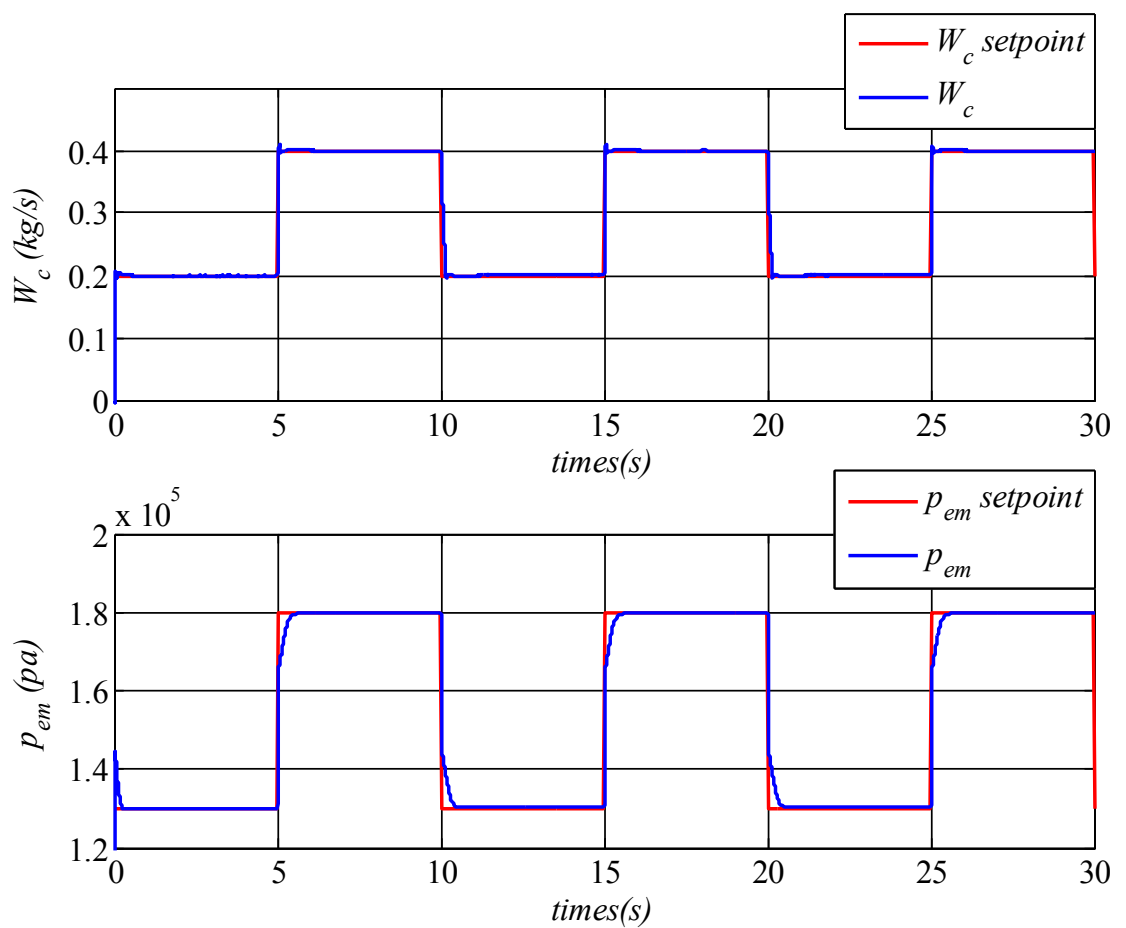

Figure 5. Control variables Wc and pem. 
The response of related variable $p_{i m}$, the intake manifold pressure, is displayed in Figure 6. From equation (15), the desired $p_{i m d}$ is calculated by $W_{c d}$ and $P_{c}$, for the variable $P_{c}$ (Refer to Figure 7) can be changed with the engine speed $\omega$, $p_{\text {imd }}$ has a little change when engine speed changes a lot.

Referring to (29), the total disturbances can be written as

$$
\dot{S}=-\gamma S-\lambda \operatorname{sgn} S-E_{2}
$$

Then, the disturbances $d_{1}, d_{2}$ and their estimation $z_{21}, z_{22}$ are obtained and shown in Figure 8.

Figure 8 indicated that the designed ESO can estimate the total disturbances and the system can still reject it even if there exists some severe changes.

And the designed control laws $u_{1}, u_{2}, u_{3}$ are shown in Figure 9. As can be seen from Figure 9, the control law $u_{1}$ which designed via a Lyapunov function based controller, that is the fuel mass flow rate $W_{f}$, is always a positive variable. In air-path subsystem, the curves of related control laws $u_{2}$ and $u_{3}$ are relatively smooth, and the common chattering problem in sliding mode control is inhibited well, which also verifies the effectiveness of alleviating the chattering problems of the designed method using exponential reaching law and ESO.

\section{Conclusion}

In this paper, a fourth-order simplified model of the air and speed-path two loop system which contains actuator faults is established. We design a controller based on Lyapunov function for the speed-path and a sliding mode controller based on exponential reaching law for the airpath. An ESO is added in to estimate and compensate the total disturbances. The simulation results show that the sliding mode controller which utilizes observer and reaching law can alleviate the chattering problem obviously and the designed two loop dynamic

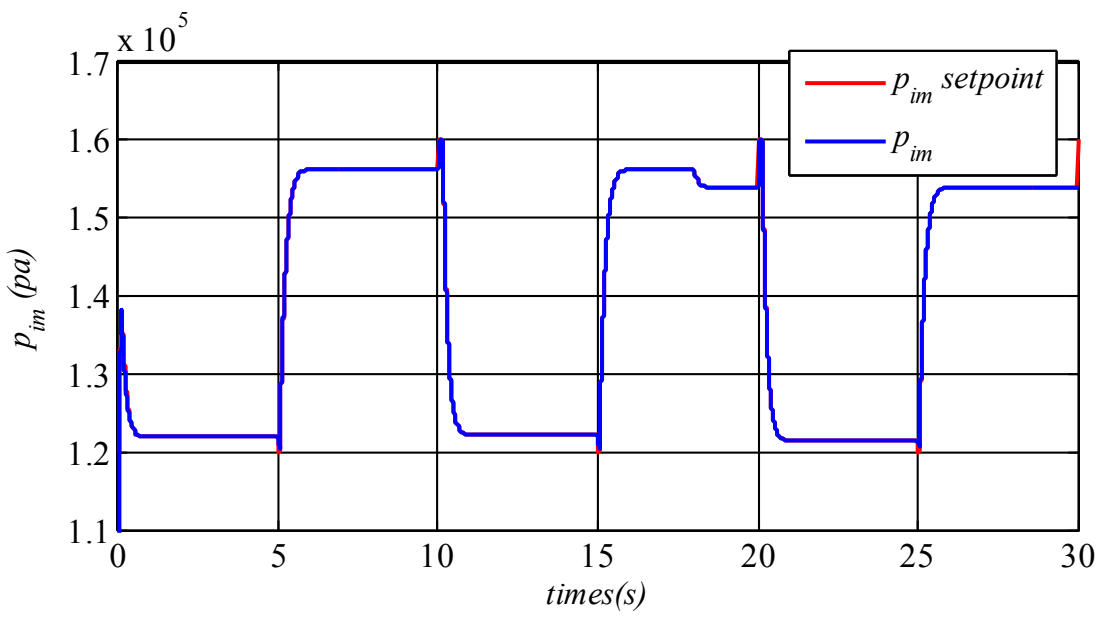

Figure 6. Intake manifold pressure pim.

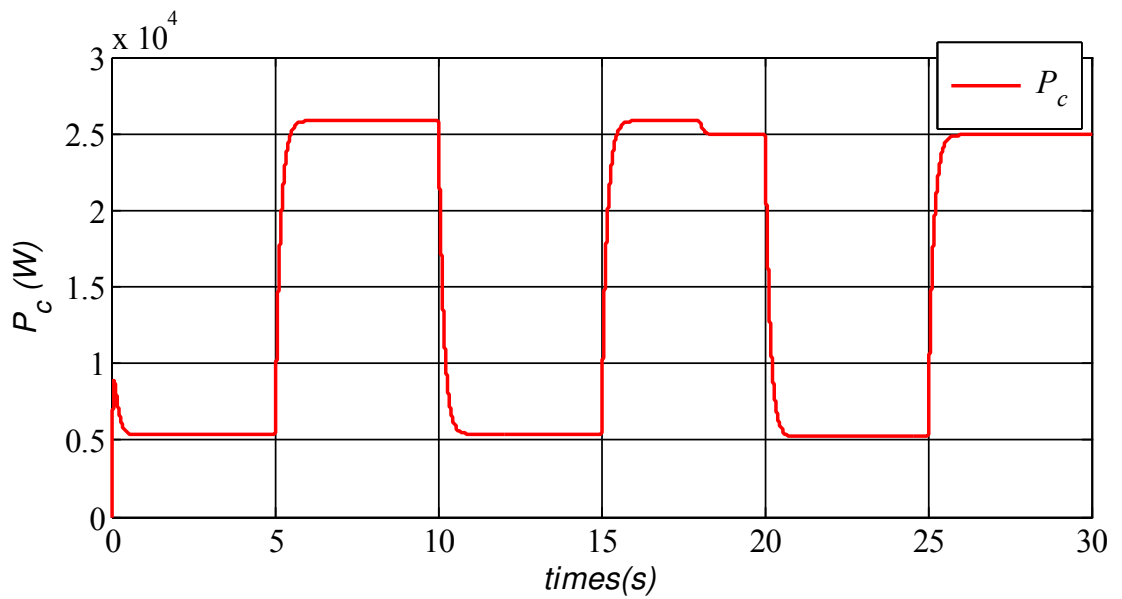

Figure 7. Compressor power $P_{c}$. 

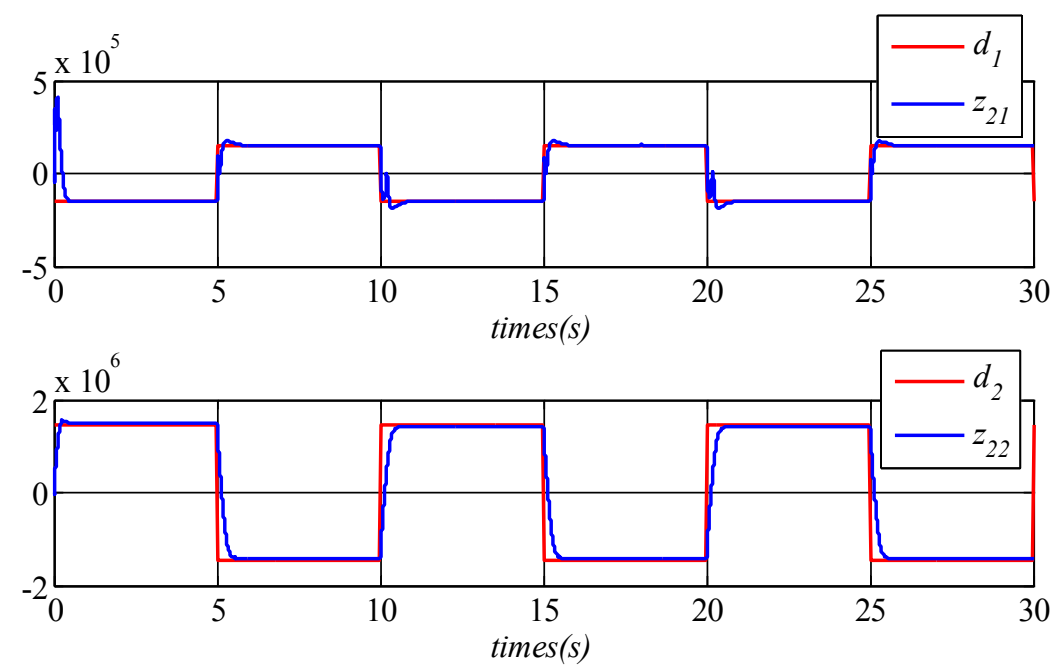

Figure 8. Estimation of disturbances via ESO.
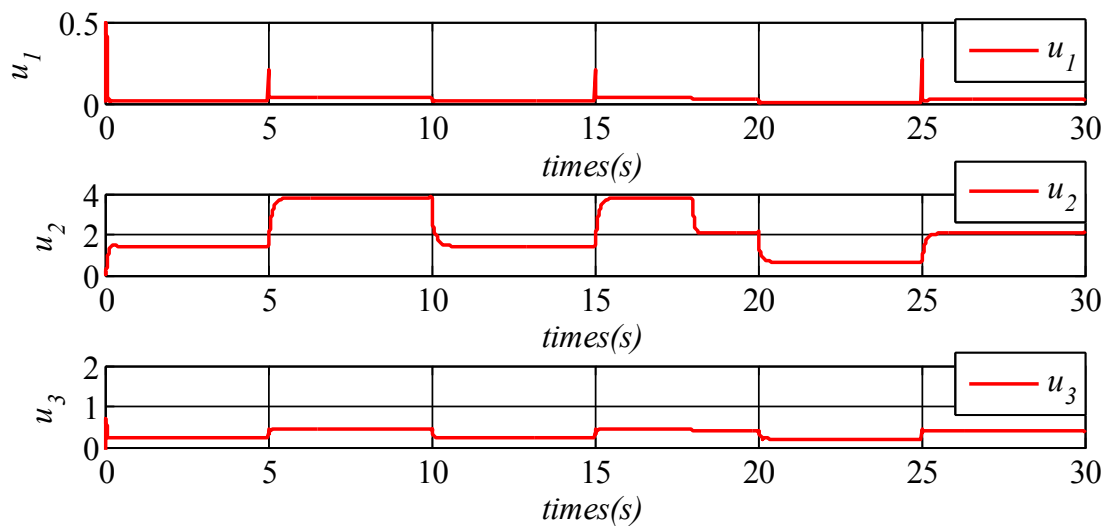

Figure 9. Control laws u1, u2 and u3.

system has good tracking performance and immunity performance.

\section{Acknowledgement}

This work was partially supported by the National Natural Science Foundation of China (61304077, 61203115), by International Science \& Technology Cooperation Program of China (2015DFA01710), by the Natural Science Foundation of Jiangsu Province (BK20130765), by the Specialized Research Fund for the Doctoral Program of Higher Education of China (20123219120038), by the Chinese Ministry of Education Project of Humanities and Social Sciences (13YJCZH171), by the 11th Jiangsu Province Six talent peaks of high level talents (2014_ZBZZ_005), by the Fundamental Research Funds for the Central Universities (30920130111014), and by the Zijin Intelligent
Program of Nanjing Univ. Sci. and Technol. (2013_ZJ_0105).

\section{REFERENCES}

1. CABRERA, E., G. CABRERA, B. CRAWFORD, F. PAREDES, J. M. RUBIO, R. SOTO, A Hybrid Approach Using an Artificial Bee Algorithm with Mixed Integer Programming Applied to a Large-Scale Capacitated Facility Location Problem, Mathematical Problems in Engineering, vol. 2012, 2012.

2. GLOVER, F., M. LAGUNA, Tabu Search, Norwell, MA, USA: Kluwer Academic Publishers, 1997.

3. HOOS, H., Automated Algorithm Configuration and Parameter Tuning, in Autonomous Search, Y. Hamadi, E. Monfroy, and F. Saubion, Eds. Springer Berlin Heidelberg, 2012, pp. 37-71. 
4. GONZÁLEZ-HIDALGO, M., A. JAUMEI-CAPÓ, A. MESEJO-CHIONG, O. L. VERA-PÉREZ, Automatic Parameter Configuration: A Case Study on a Rehabilitation Oriented Human Limb Tracking Algorithm, Studies in Informatics and Control, vol. 23, no. 3, 2014, pp. 313-323.

5. KOBAYASHI, R., N. KOMODA, M. MORI, M. SAMEJIMA, Cost-benefit Analysis of Decentralized Ordering on Multitier Supply Chain by Risk Simulator, Studies in Informatics and Control, vol. 21, no. 1, 2012, pp. 75-83.

6. CRAWFORD, B., E. MONFROY, F. PAREDES, R. SOTO, C. VALENZUELA, Parameter Tuning of Metaheuristics Using Metaheuristics, Advanced Science Letters, vol. 19(12), 2013, pp. 3556-3559.

7. KOZA, J., Genetic Programming as a Means For Programming Computers by Natural Selection. Kluwer Academic Publishers, vol. 4, no. 2, 1994.

8. BEASLEY, J., An Algorithm for Solving Large Capacitated Warehouse Location Problems, European Journal of Operational Research, vol. 33, no. 3, 1998, pp. 314-325.

9. BÖLTE, A., U. W. THONEMANN, Optimizing Simulated Annealing Schedules with Genetic Programming, European Journal of Operational Research, vol. 92, no. 2, 1996, pp. 402-416.

10. KIRKPATRICK, S., Optimization by Simulated Annealing: Quantitative Studies. Journal of Statistical Physics, vol. 34, no. 5-6, 1984, pp. 975-986.

11. GELATT, C. D., S. KIRKPATRICK, M. P. VECCHI, Optimization by Simulated Annealing, Science, vol. 220, no. 4598, 1983, pp. 671-680.

12. METROPOLIS, N., A. W. ROSENBLUTH, M. N. ROSENBLUTH, A. H. TELLER, E. TELLER, Equation of State Calculations by Fast Computing Machines, The Journal of Chemical Physics, vol. 21(6), 1953, pp. 1087-1092.

13. CABRERA, G., C. CUBillos, J. P. RIQUELME, S. RONCAGLIOLO, R. SOTO, A Hybrid Particle Swarm Optimization-Simulated Annealing
Algorithm for the Probabilistic Travelling Salesman Problem, Studies in Informatics and Control, vol. 21, 2012, pp. 49-58.

14. CHENG, Z., C. C. KANG, W. C. LIN, S. LIU, W. H. WU, A Single-Machine TwoAgent Scheduling Problem by a BranchAnd-Bound and Three Simulated Annealing Algorithms, Discrete Dynamics in Nature and Society, vol. 2015, 2015, Article ID 681854.

15. NI, L., J. QIN, H. XIANG, Y. YE, A Simulated Annealing Methodology to Multiproduct Capacitated Facility Location with Stochastic Demand, The Scientific World Journal, vol. 2015, 2015, Article ID 826363.

16. LUNDY, M., A. MESS, Convergence of an Annealing Algorithm. Mathematical Programming, vol. 34, 1986, pp. 111-124.

17. WILHELM, M., T. WARD, Solving Quadratic Assignment Problems by Simulated Annealing, IIE Transactions, vol. 19, no. 1, 1987, pp. 107-119.

18. FOGEL, L. J., A. J. OWENS, M. J. WALSH, Artificial Intelligence through Simulated Evolution, New York, USA: John Wiley, 1966.

19. HOLLAND, J. H., Adaptation in Natural and Artificial Systems, Ann Arbor, MI, USA: University of Michigan Press, 1975.

20. RECHENBERG, I., Evolutionsstrategie: Optimierung Technischer Systeme nach Prinzipien der Biologischen Evolution, Ph.D. dissertation, TU Berlin, 1971.

21. EGgermont, J., Data Mining Using Genetic Programming: Classification and Symbolic Regression, Ph.D. dissertation, Universiteit Leiden, September 2005.

22. WALKER, M., Introduction to Genetic Programming, Computer Science Department, Montana State University, Tech. Rep., December 2001.

23. BEASLEY, J., OR-Library: Distributing Test Problems by Electronic Mail, Journal of the Operational Research Society, vol. 41(11), 1990, pp. 1069-1072. 